\title{
Presentation Entanglemen States and its Application in Quantum Computation
}

\section{YADOLLAH FARAHMAND ${ }^{1,4 *}$, ZABIALAH HEIDARNEZHAD², FATEMEH HEYDARNEZHAD ${ }^{3}$, KH.KH. MUMINOV ${ }^{1}$ and SEYEDEH ZEINAB HOSSEINIRAD ${ }^{5}$}

\author{
${ }^{1}$ Physical-Technical Institute named after S.S.Umarov Academy of Sciences ,Dushanbe, Tajikistan. \\ ${ }^{2}$ Young Researchers Club, Andimeshk Branch, Islamic Azad University, Andimeshk, Iran. \\ ${ }^{3}$ Department of Chemistry,Andimeshk Branch, Islamic Azad University, Andimeshk, Iran. \\ ${ }^{4}$ Farhangiyan Golestan University, Iran. \\ ${ }^{5}$ Farhangiyan Mazandaran University, Iran. \\ ${ }^{*}$ Corresponding author E-mail: amirfrhmnd@gmail.com
}

http://dx.doi.org/10.13005/ojc/300257

(Received: March 03, 2014; Accepted: March 04, 2014)

\begin{abstract}
In this article we will consider the entanglement states and we will formulate the density matrix and the expectation value of entanglement state to mathematics and also we will explain the applications of entanglement states in superdense coding and sending information ( qubits transfer).
\end{abstract}

Key words: Quantum entanglement states, Density matrix, Expectation value, Super_dense coding, Quantum teleportation.

\section{INTRODUCTION}

In quantum mechanics theory we can see the entanglement phenomena which are include two particles( photon or electron) in a separated space but their properties are related together in some way, at first this kind of system was interduced by Einstein_Podolski and Rosen( EPR)1-3 such as pair particle that each of them have spin $1 / 2$ and total zero spin that have anqular momentum total zero. The information(qubits) are transferred by intertwining phenomena of two objects quantum states. In other word entanglement phenomena of two objects quantum states can physically be in separated places, but have shared information ( qubits) in a same time. Before researchers had been succeed to intertwine the pairs of spin quantum states of electrons and the pair of spin quantum states of electrons and etc, by the way it is a unexpected and beyond conception phenomena in quantum theory mechanic.

\section{Entanglement states}

If we exert the following Git to the two qubit, the out let qubit is the result of the entanglement qubit. 


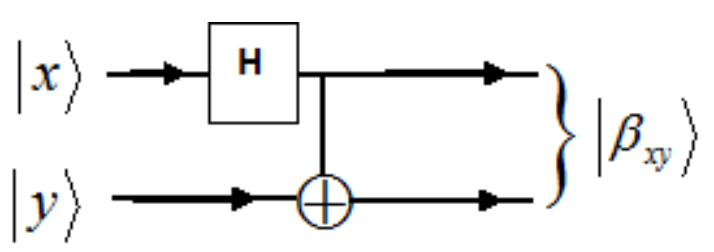

Out let in up orbit for $\mathrm{x}=\mathrm{y}=0$ is the qubit state.

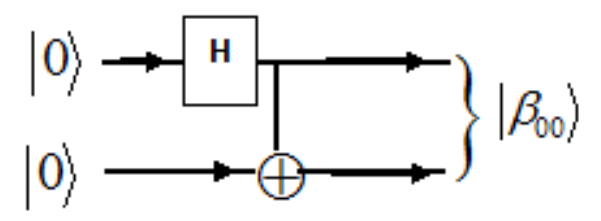

and

$$
\left|\beta_{00}\right\rangle=\frac{1}{\sqrt{2}}(|00\rangle+|11\rangle
$$

\section{Calculation}

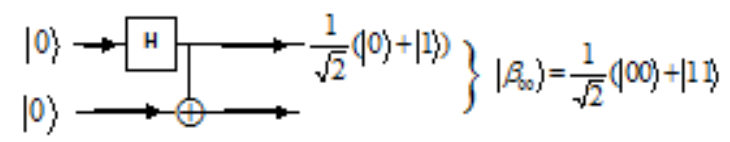

$$
H|0\rangle-\frac{1}{\sqrt{2}}\left(\begin{array}{cc}
1 & 1 \\
1 & -1
\end{array}\right)\left(\begin{array}{l}
1 \\
0
\end{array}\right)-\frac{1}{\sqrt{2}}\left(\begin{array}{l}
1 \\
1
\end{array}\right)-\frac{1}{\sqrt{2}}\left[\left(\begin{array}{l}
1 \\
0
\end{array}\right)+\left(\begin{array}{l}
0 \\
1
\end{array}\right)\right]-\frac{1}{\sqrt{2}}(|0\rangle+|1\rangle)
$$$$
|0\rangle \oplus|0\rangle=|0\rangle \quad, \quad|1\rangle \oplus|0\rangle=|1\rangle
$$$$
\left.\frac{1}{\sqrt{2}}(|0\rangle+|1\rangle)|0\rangle \underset{(c-\text { nor }) \text { gat }}{\longrightarrow} \frac{1}{\sqrt{2}}(00\rangle+|11\rangle\right)
$$

This state can't be shown by two isolate qubits; and there is a correlation between two qubits, the name of this qubits is entanglement. Even in so far distance this correlation between two qubits don't be separated from each other.

This kind of correlation is known as John.Bell non-local correlation and it's a response for Podox[batelnama] EPR .

The similar states of entanglement and correlation sates of two qubits that are known as
EPR or BEL4-8 are:

$$
\begin{aligned}
& \left|\beta_{01}\right\rangle=\frac{1}{\sqrt{2}}(|01\rangle+|10\rangle) \\
& \left|\beta_{10}\right\rangle=\frac{1}{\sqrt{2}}(|00\rangle-|11\rangle) \\
& \left|\beta_{11}\right\rangle=\frac{1}{\sqrt{2}}(|01\rangle-|10\rangle)
\end{aligned}
$$

The simple model of quantum entanglement state The mention the simple model of quantum entanglement state we can consider to a double electron system that just have spin. If we show the first spin with S_1 and the second spin with S_2, we can attribute a total spin $\mathrm{S}$ to the this system.

$$
\begin{aligned}
& S=S_{1}+S_{2} \\
& {\left[S_{i x}, S_{i y}\right]=i \varepsilon_{x y z} \hbar S_{i z}(2)}
\end{aligned}
$$

The total S size is achieved from following relation 9 .

$$
\begin{aligned}
& \left|S_{1}-S_{2}\right| \leq S \leq\left(S_{1}+S_{2}\right) \\
& \text { Example: } S_{1}=\frac{1}{2} ; S_{2}=\frac{1}{2} \\
& \left\{\begin{array}{l}
|\uparrow \uparrow\rangle \\
\sqrt{2}(|\uparrow \downarrow\rangle+\mid \downarrow \uparrow\}) \quad * \quad \text { Triplet (3) } \\
\frac{1}{\sqrt{2}}(|\uparrow \downarrow\rangle-|\downarrow \uparrow\rangle) \quad * \quad \text { Singlet (4) }
\end{array}\right.
\end{aligned}
$$

The bipartite quantum system ( tow qubit)

we can find the wonderful of quantum mechanic by checking the properties of two qubits quantum states. \{?|0??_A,?|1??_A \}, $\{? \mid 0 ? ?$ B,?|1??_B $\}$ are respectively the representation of orthonormal bases $A$ and $B$, we image that two qubits quantum states is like the following form.

$|\psi\rangle_{A B}=a|0\rangle_{A} \otimes|0\rangle_{B}+b|1\rangle_{A} \otimes|1\rangle_{B}(5)$ 
In this case qubit $\mathrm{A}$ and $\mathrm{B}$ are correlate in regard to each other.

The measurement of qubit $A$ by image on bases $\{$ ?|0??_A,?|1??_B $\}$ with probability la|^2 give the result ?I0??_A and it make appear the measurement of state of $\{$ ?|0??_B,?|1??_B $\}$ and with probability $|\mathrm{|b}|^{\wedge} 2$ give the result ?|1??_A and make appear the state of ?|0??_B ?|1??_B ; so if we achieve the results of ?|1??_A,?|0??_A, we can find the result ?|1??_B, ?|0??_B with insure ( with one probability).

similarly with measurement the qubit $B$ we achieve the similar results. It mean the out lets of $\{$ ?|0??_A,?|1??_A $\},\{?|0 ? ?, B, ?| 1$ ??_B $\}$ that come from measurements in ?I???_AB state are completed correlative.

It mean the outlets of $\left\{?\left|0 ? ? \_A, ?\right| 1 ? ? \_A\right\}$ , \{?|0??_B,?|1??_B $\}$ that come from measurement in ?I???_AB state are completely correlative.

Two qubits state ?|???_AB discussion is propounded for the bipartite quantum system ( Non division to two part).

Hilbert space of bipartite system H_A H_B.

In total state, the pure state of bipartite can be shown like following form:

$$
\begin{aligned}
|\psi\rangle_{A B} & =\sum_{i \mu} a_{i \mu}|i\rangle_{A} \otimes|\mu\rangle_{B} \\
\sum_{i \mu} a_{i \mu} & =1
\end{aligned}
$$

The physics model of entanglement qubit at pair production and pair annihilation:(Figure 1)

\section{Expectation value calculation of observable $M \_A$ on?|???_AB bipartite pure state}

By regard to observable as $M \_A$ I_A that $M \_A$ operator itself is adjunctive that is exerted on A and I_B operator is unit is exerted on B.

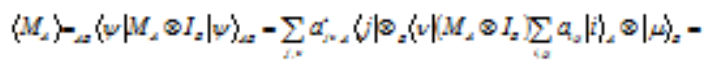

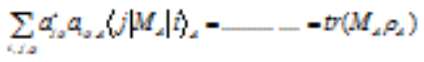

By regard to former calculations for single qubit and by regard to the following relation for matrix density we have :

Example:

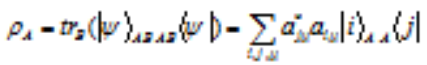

$$
\begin{aligned}
& |\psi\rangle_{\lambda s}-a|0\rangle_{A} \otimes|0\rangle_{s}+b|1\rangle_{A} \otimes|1\rangle_{s} \\
& \left\langle M_{A}\right\rangle_{A}\left\{\left\langle\left|M_{A} \odot I_{z}\right| \psi\right\rangle_{A s}-\right.
\end{aligned}
$$

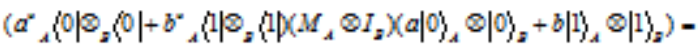

$$
\begin{aligned}
& -|a|^{2}\left\langle\left(\left|M_{A}\right| 0\right\rangle_{A}+|8|^{2}\left(1\left|M_{A}\right|\right\rangle_{A}-\operatorname{tr}\left(M_{A} \rho_{A}\right)\right.
\end{aligned}
$$

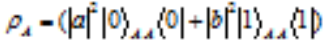

\section{The applications of entanglement state}

In some methods that the intertwining process can lay for purpose of application use, we will consider to two cases of that 10-12:

\section{Super-dense coding}

Let us assume that Alice at Amsterdam wants to send two bits of sending two separated bits. It is a question that can according to quantum mechanic theory for sending that, implement just by a single qubit? The answer is yes, in this field there are a lot of studies that coding the double bit classical massage in a pair of entanglementparticle( one qubit ). So on codition that Alice and Bob have been shared the qubit together at state of Bell(Figure 2), they say I?_00?.

If this qubit that must be sent is 00 , as a result Alice simply would send her qubit to Bob that now have qubit in the state of I?_00?.

If a qubit which must be sent is 01 , as a result Alice would use a quantum Git NOT $X$ for her qubit ( assume that the first member of this pair in Bel state 1?_01?. Alice similary use the suit transformation for her qubit. The consider qubits which must be sent, can be to the form of other combination from 10 mand 11 that is sent to Bob.

The total result is that classical two bit $x y$ would be coded by a single Bel statel?_xy?. This kind of coding is created from a number of classical bits single entanglement quantum state, is known as a extremely mass coding. Where as four state of Bel are Orthonormal, consequently, certainly they 
are recognizable by a suitable measurement. So Bob can decode the qubitentanglement state that is in his hand and he can achieve the classical two bits information.(Figure 3)

If the entanglementqubit be in the form of I?_00? , Alice will not do anything on that for sending that; But if two entanglementqubit be in other form like I?_01?, I?_10?, I?_11? and Alice wants to send that to Bob, she must do the following actions for each one.

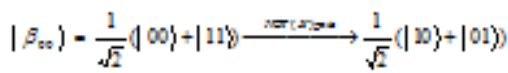

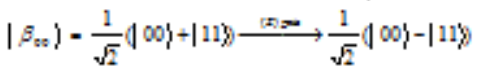

$$
\begin{aligned}
& \left.\left.\mid \beta_{80}\right)=\frac{1}{\sqrt{2}}(\mid 00)+|11\rangle\right) \longrightarrow \frac{1}{\sqrt{2}}(01\rangle-\mid 100
\end{aligned}
$$

In this case, according to the (Figure 3) Alice would coding classical two bits in the entanglementqubit. Then Bob would decode by measurement on classical two bits qubit.

Let us assume that Alice and Bob are share in entanglementqubit of $\left.\left|\beta_{10}\right\rangle=\frac{1}{\sqrt{2}}(10\rangle-|01\rangle\right)$

That the first qubitis for Alice and the second qubit is for Bob. If Bob achieve to qubit 10 ? by measurement on entanglement state, this mean is that he decode the classical $B i t y=0$. and by knowing the entanglementqubit could get a result that $x=1$, so with a measurement by Bob the classical bits in entanglementqubit would be decoding .

\section{Quantum Teleportation}

Alice in Amsterdam wants to send a desirable mono qubit $|\psi\rangle=\alpha|0\rangle+\beta|1\rangle$ to Bob in Boston. She can't determine this state and send that information to Bob. Unless again we image that Alice and Bob have shared at a pair of intertwindqubits in one of the Bel state, such as:

$$
\left|\beta_{00}\right\rangle=\frac{1}{\sqrt{2}}(|00\rangle+|11\rangle)
$$

The qubit that must be sent with entanglementstate(pair) from far distance is started from three qubits state.

$\left.\left.\left.\left|\psi_{0}\right\rangle=|\psi\rangle \beta_{\infty}\right\rangle=\frac{1}{\sqrt{2}}(\alpha|0\rangle(00\rangle+|11\rangle)+\beta|1\rangle|00\rangle+|11\rangle\right)\right)$

Here, normally, two firstqubit are in company with Alice and the third qubit is in company with Bob.(Figure 4)

Now Alice lay her two qubit throughout a quantum git C-NOT. This process capture the qubit that must be sent from far distance in company with a part of entanglement state which is belong to Alice and it would pair them.

Of course this entanglement state is captured for creating and starting the work. As a result this three qubits in the state of captured (entanglement) would end to:

$\left.\left.\left|\psi_{1}\right\rangle=\frac{1}{\sqrt{2}}[\alpha|0\rangle(|00\rangle+|11\rangle)+\beta|1\rangle|| 10\rangle+|01\rangle\right)\right]_{(13)}$

With regard to mentioned models in (1-3-8-1) and (2-3-8-1) the up word relation is achieved.

Then Alice would send the first qubit in throughout a Hadamardgit and we have our two qubits of calculation with application of Hadamardgit on qubit $|\psi\rangle$ :

$$
\begin{aligned}
& H|\psi\rangle-H(\alpha|0\rangle+\beta|1\rangle)-\alpha\left(\frac{1}{\sqrt{2}}(|0\rangle+|1\rangle)+\beta\left(\frac{1}{\sqrt{2}}(|0\rangle-|1\rangle)\right.\right. \\
& -\frac{1}{\sqrt{2}}[\alpha(|0\rangle+|1\rangle)+\beta(|0\rangle-|1\rangle]
\end{aligned}
$$

$\left.\left|\psi_{2}\right\rangle=\frac{1}{2}[\alpha(|0\rangle+|1\rangle)(|00\rangle+|11\rangle)+\beta(0\rangle-|1\rangle)|10\rangle+|01\rangle\right]$

With a simple Tensory product operation and transfer the coefficient ?,? to the third qubit we have:

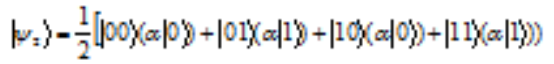

$$
\begin{aligned}
& +\mid 01 y(\beta \mid 0)+100(\text { (f11)-|11)( }(10)-\mid 10 y(\beta \mid 1)] \\
& -\frac{1}{2}[100\rangle(a|0\rangle+\beta|1\rangle)+|01\rangle(a|1\rangle+\beta|0\rangle) \\
& +|10(\alpha \mid 0)-\beta| 1\rangle+|11 Y(\alpha|1\rangle-\beta|0\rangle)]
\end{aligned}
$$


So, the result of Alice's measurement with probability of 1 ? 4 will be one of the pairs $11,10,01,00$ and the state that is according to that in each Bob's gubits that will be in left side is like this from:

$$
\begin{aligned}
& 00 \rightarrow\left|\psi_{00}\right\rangle=\alpha|0\rangle+\beta|1\rangle \\
& 01 \rightarrow\left|\psi_{01}\right\rangle=\alpha|1\rangle+\beta|0\rangle \\
& 10 \rightarrow\left|\psi_{10}\right\rangle=\alpha|0\rangle-\beta|1\rangle \\
& 11 \rightarrow\left|\psi_{11}\right\rangle=\alpha|1\rangle-\beta|0\rangle
\end{aligned}
$$

Now if Alice say her result to Bob (on a classical chanel like telephone or email or a quantum chanel like so mass coding), Bob will understand the circumstance of deformation and his qubit state transformation to $|\psi\rangle$. If the result is 00 , Bob will not do anything, because now he is in state $|\psi\rangle$. For other probability results, Bob must use a suitable combination of $Z$ and $X$. If the result be $m_{-} 1 \mathrm{~m} \_2$, so the total result will be like this from(Figure 5).

For example : if Alice achieve the qubit $\left|\psi_{01}\right\rangle$ in her measurement, by sending a massage to Bob (classical two bit) she will inform him that a qubit that is sent to him is $\left|\psi_{01}\right\rangle=\alpha|1\rangle+\beta|0\rangle$, Bob by using the suitable Git i.e. Git $\operatorname{NOT}(X)$ will receive that qubit.

$$
\left.X\left|\psi_{01}\right\rangle=X \alpha 1\right\rangle+X \beta|0\rangle=\alpha X|1\rangle+\beta X|0\rangle=\alpha|0\rangle+\beta|1\rangle \ldots
$$

It's like a magic in quantum mechanic. By the way this problem have been achieved practically at laboratory by sending a ray or photon light from one room to another room.

\section{There is a point:}

Sending process from far distance don't contradict the noclonictheory.i.e. copy of a desirable unknown state don't be accomplished, but the main state in current process would have a little deformation.

Sending process from far distance don't contradict the special relativity theory, in this basis inevitable the practical information physically establish connection with a speed that is less than light.

\section{CONCLUSION}

Intertwining is one of the sources of establishment of secret quantum connections. This phenomena say that if two bits entanglement with each other, if they be far a from each other even millions kilometers and upon one of we perform the measurement process, at that moment we can achieve the information of the second bit. They have effect on each other, i.e. higher than light speed and immediately! Einstein called this subject as a ghostly process in far distance that is like to a connection between two entanglement pieces by invisible wires which we don't have any information from them, but it is a total principle for quantum computations. Scientists like Einstein, Podloski and Rosen introduced the EPR theory.

Surprising phenomena of intertwining in quantum mechanic theory made a big evolution in quantum theory and also in quantum computers; inaddition to mentioned applications in this chapter, intertwining phenomena has a so attractive application in quantum computers i.e. it has a secret quantum key distribution that its importance is not secret ton anyone. One of the so important result of intertwining phenomena is that it contradict the locality and it proved that the nature of quantum mechanic theory is non-local. 


\section{REFERENCES}

1. Perskill, J. Lecture notes for Ph219/CS219:

7. Auletta, G.. New Jersey. London. Hong Kong. Chapter 4, Quantum Information and computation. 2001

2. Auletta, G. New Jersey. London. Hong Kong. 2001; 531-544

8. Perskill, J. Lecture notes for Ph219/CS219:

9. Sakurai, J.J. Modern Quantum Mechanics, Chapter 2, Foundations 1: State and Ensembles, 2001.
3. Prers, A. Quantum Theory,Newyork, Boston, London, Moscow. 2002; 148-155

4. Sara, M.McMurry. Quantum Mechanics.1993, 337-346

5. Zeilinger, A. A Fundamental Concept Finding its Applications .1997, 12-18

6. Auletta, G. New Jersey. London. Hong Kong. 2001: $42-44$

\section{2}

10. Chatterjee, A. Introduction to quantum computation.quant-ph/0312111, 2003

11. Auletta, G. World scientific, Singapore. New Jersey. London. Hong Kong. 2001; 743-755

12. Michael A. N.; Isaac, L. C. World scientific, Singapore. 2003; 26-28. 